\title{
Organic Cation Transporter 1 (OCT1) mRNA expression in hepatocellular carcinoma as a biomarker for sorafenib treatment
}

\author{
Daniel Grimm¹, Jonas Lieb', Veronika Weyer², Johanna Vollmar', Felix Darstein', Anja Lautem³, \\ Maria Hoppe-Lotichius ${ }^{3}$, Sandra Koch ${ }^{1}$, Arno Schad ${ }^{4}$, Jörn M. Schattenberg ${ }^{1}$, Marcus A. Wörns ${ }^{1}$, Arndt Weinmann ${ }^{1}$, \\ Peter R. Galle ${ }^{1}$ and Tim Zimmermann ${ }^{1 *}$
}

\begin{abstract}
Background: The polyspecific organ cation transporter 1 (OCT1) is one of the most important active influx pumps for drugs like the kinase inhibitor sorafenib. The aim of this retrospective study was the definition of the role of intratumoral OCT1 mRNA expression in hepatocellular carcinoma (HCC) as a biomarker in systemic treatment with sorafenib.

Methods: OCT1 mRNA expression levels were determined in biopsies from 60 primary human HCC by real time PCR. The data was retrospectively correlated with clinical parameters.

Results: Intratumoral OCT1 mRNA expression is a significant positive prognostic factor for patients treated with sorafenib according to Cox regression analysis (HR 0.653, $95 \%-\mathrm{Cl} 0.430-0.992 ; p=0.046$ ). Under treatment with sorafenib, a survival benefit could be shown using the lower quartile of intratumoral OCT1 expression as a cut-off. Macrovascular invasion (MVI) was slightly more frequent in patients with low OCT1 mRNA expression ( $p=0.037)$. Treatment-induced AFP response was not associated with intratumoral OCT1 mRNA expression levels $(p=0.633)$.
\end{abstract}

Conclusions: This study indicates a promising role for intratumoral OCT1 mRNA expression as a prognostic biomarker in therapeutic algorithms in HCC. Further prospective studies are warranted on this topic.

Keywords: Hepatocellular carcinoma, HCC, OCT1, SLC22A1, Biomarker, Sorafenib

\section{Background}

Hepatocellular carcinoma (HCC) belongs to the most common human cancer entities and shows an increasing incidence $[1,2]$. With an estimated 5-year-survival rate of $15 \%$ the prognosis of HCC patients is poor [3]. Curative treatment options are only available for early tumor stages. In particular, patients with a multifocal tumor growth are facing a poor prognosis. Classical chemotherapeutic approaches are largely inefficient due to a pronounced chemoresistance [4]. To date, the oral multikinase inhibitor sorafenib is the standard systemic treatment for patients with advanced HCC [2]. The SHARP trial showed an increase in the median overall

\footnotetext{
* Correspondence: tim.zimmermann@unimedizin-mainz.de

${ }^{1}$ 1st Department of Medicine, University Medical Center Mainz,

Langenbeckstr. 1, Mainz 55131, Germany

Full list of author information is available at the end of the article
}

survival of about 3 months in the sorafenib treatment group [5]. The effects of sorafenib were slightly weaker in a phase III trial in an asia-pacific population with a more advanced disease [6]. Unfortunately, a substantial fraction of patients faces serious drug-related adverse events under sorafenib treatment that can even result in drug discontinuation. Diarrhea and hand-foot skin reaction are the most common reactions and occur in about 8-16\% [5, 6]. Moreover, there are controversial assumptions regarding the cost effectiveness of sorafenib treatment $[7,8]$. These findings underscore the urgent need for biomarkers predicting prognosis and response under treatment with sorafenib. However, convincing biomarkers for the identification of patients that will most likely have a benefit from a systemic treatment with sorafenib are still not defined [9]. 
The organic cation transporter OCT1 (gene symbol SLC22A1) belongs to the amphiphilic solute facilitator (ASF) family of integral transmembrane proteins [10]. It is located at the basolateral membrane of hepatocytes [11]. The physiologic role of OCT1 is the uptake of a broad range of endogenous (e. g. catecholamines and prostaglandins) and exogenous substrates including anticancer drugs like tyrosine kinase inhibitors (e. g. sorafenib) [11-13]. We could show previously that intratumoral downregulation of OCT1 correlates with a worse survival in HCC [10]. In addition, a high pretherapeutic OCT1 expression predicts a complete molecular response to the tyrosine kinase inhibitor imatinib in chronic myeloid leukemia (CML) [14]. It is known that a reduced or aberrant OCT1 expression prevents a sufficient intracellular sorafenib concentration [13].

It was the aim of this retrospective study to define whether OCT1 mRNA expression is a useful biomarker in the systemic therapy of $\mathrm{HCC}$ with sorafenib.

\section{Methods}

\section{Patient characteristics and tissue samples}

Clinical data and tumor samples of 60 patients that underwent liver biopsy at the University Medical Center Mainz between January 2001 and December 2013 were analyzed in this study. Clinical and pathological characteristics of this cohort are summarized in Table 1. Primary inclusion criteria were liver biopsy, treatment with sorafenib and registration in the HCC database Mainz. Main exclusion criteria were insufficient RNA-extraction from liver tissue and curative liver transplantation without post-transplant tumor recurrence. All HCC were histologically confirmed. This study was approved by the ethics committee of the local medical board RhinelandPalatinate and was conducted according to the ethical guidelines of the Declaration of Helsinki. Written informed consent was given by each patient. The liver tissues analyzed in this study were embedded in paraffin. For the evaluation of an AFP response, only patients with AFP levels > $20 \mathrm{ng} / \mathrm{ml}$ (AFP-positive HCC) were included. Due to the retrospective approach, AFP response was determined individually at variable time points after initiation of sorafenib treatment.

\section{RNA isolation, RT-PCR and real-time RT-PCR analysis}

Paraffin embedded tissue sections of 5-10 $\mu \mathrm{m}$ thickness were used for RNA isolation. Hemo-De solvent (Scientific Safety Solvents, Keller, USA) and the High Pure RNA Paraffin Kit (Roche, Mannheim, Germany) were used for deparaffinization according to the manufacturer's recommendations. The iScript cDNA Synthesis kit (Biorad, Munich, Germany) was applied for cDNA synthesis from total RNA according to the manufacturer's recommendations. Quantification of OCT1
(SLC22A1) transcripts was performed by real-time PCR. Quantitect SYBR Green PCR Kit (QIAGEN, Hilden, Germany) and validated primers of a Quantitect Primer Assay with the primer sets Hs_SLC22A1_1_SG (QT00019572) and Hs_GAPDH_2_SG (QT01192646) were used according to the manufacturer's recommendations (QIAGEN, Hilden, Germany). Primer sequences are considered commercially sensitive by the manufacturer and cannot be published. For the amplification, an initial denaturation $\left(15 \mathrm{~min}\right.$ at $95^{\circ} \mathrm{C}$ ) followed by $50 \mathrm{cy}$ cles of denaturation $\left(15 \mathrm{~s}\right.$ at $\left.94{ }^{\circ} \mathrm{C}\right)$, annealing $(30 \mathrm{~s}$ at $55{ }^{\circ} \mathrm{C}$ ), and elongation $\left(30 \mathrm{~s}\right.$ at $\left.72{ }^{\circ} \mathrm{C}\right)$. A LightCycler ${ }^{\circ}$ 480 real-time PCR system (Roche, Mannheim, Germany) was used. Relative expression level of OCT1 (SLC22A1) was calculated by normalization to GAPDH gene expression using LightCycler ${ }^{\circledR} 480$ software version 1.5.0.

\section{Statistical analysis}

Statistical analyses were performed using SPSS (IBM ${ }^{\bullet} \mathrm{SPSS}^{\curvearrowleft}$ 21 version 21.0.0.1). For descriptive analyses, mean and standard deviation were calculated for continuous variables. In addition, absolute and relative frequencies were computed for categorical variables. Quantitative, normally distributed variables were analyzed using the unpaired t-test. For the analysis of categorical variables, we used Fisher's exact test or Mann-Whitney U test. Survival rates between both OCT1 groups were compared by the log-rank test. For graphical visualization Kaplan-Meier curves are presented. The univariable test results have to be considered as explorative. No adjustments for multiple testing have been done here. $P$-values are given for descriptive reasons only. A multivariable Cox regression model adjusted for age was performed for confirmatory analysis with a significance level of $5 \%$. Hazard ratios with their corresponding $p$-values and $95 \%$ confidence limits are presented.

\section{Results}

Expression of OCT1 (SLC22A1) mRNA in HCC biopsies

First, we analyzed the intratumoral OCT1 mRNA expression levels. The relative OCT1 expression levels in HCC tissue ranged between 0.0037 and 9.711 with a lower quartile of 0.227 .

\section{Survival according to intratumoral OCT1 mRNA expression}

Cox regression analysis revealed a significant positive association between OCT1 mRNA expression level and patient survival in patients treated with sorafenib (HR 0.653; 95 \%-CI 0.430-0.992; $p=0.046$; Table 2). Patient age at beginning of sorafenib treatment did not have a significant impact $(p=0.144)$. As the majority of patients in this cohort were male, the variable gender was excluded in the cox regression analysis. A sensitivity analysis showed a slight but relevant survival benefit in the univariable log-rank test 
Table 1 Patients and tumor characteristics

Characteristics
Gender
male
female
Mean age
years (standard deviation)
Underlying disease
alcohol
HBV
HCV
steatosis or NASH
others
unknown

Prior HCC treatment

$\begin{array}{ll}\text { yes } & 35 \\ \text { no } & \\ \text { tumor grading } & \\ \text { G1 } & 13 \\ \text { G2 } & 3 \\ \text { G3 } & 8 \\ \text { unknown } & 5\end{array}$

Tumor burden

$\begin{array}{lll}\text { MVI } & \text { absent } & 31 \\ \text { EHS } & \text { present } & 29 \\ & \text { absent } & 17 \\ \text { MVI and/or EHS } & \text { present } & 43 \\ & \text { absent } & 6 \\ & \text { present } & 54\end{array}$

BCLC classification

$\begin{array}{ll}\text { A } & 1 \\ \text { B } & 1 \\ \text { C } & 50 \\ \text { D } & 6 \\ \text { unknown } & 2 \\ \text { ECOG PS } & \end{array}$

EOG

$\begin{array}{ll}0 & 11 \\ 1 & 39 \\ 2 & 5 \\ 3 & 2 \\ \text { unknown } & 3\end{array}$

Table 1 Patients and tumor characteristics (Continued)

\begin{tabular}{ll}
\hline Child-Pugh & \\
A & 14 \\
B & 24 \\
C & 4 \\
unknown & 18 \\
Ascites & \\
absent & 23 \\
present & 19 \\
unknown & 18 \\
Baseline AFP (ng/ml) & \\
$\leq 20$ & 22 \\
$>20$ & 36 \\
unknown & 2 \\
\hline
\end{tabular}

using the lower quartile of OCT1 mRNA expression as a cutoff ( $p=0.049$; Fig. 1 ). According to the sensitivity analysis, patients were subdivided into two groups by the intratumoral OCT1 mRNA expression level (<lower quartile vs. $\geq$ lower quartile, Fig. 2).

\section{OCT1 mRNA expression in correlation with patient and tumor characteristics}

Patients and tumor characteristics are listed in Table 1. No differences between the two groups (OCT1 mRNA expression $<$ lower quartile vs. $\geq$ lower quartile) could be shown regarding formerly described relevant baseline characteristics like presence of ascites $(p=0.504)$, Barcelona-Liver Cancer Clinic stage (BCLC stage, $p=0.988$ ), and Eastern Cooperative Oncology Group Performance status (ECOG; $p=0.099$, Table 3). Macrovascular invasion (MVI) was slightly more frequent in the group showing a low OCT1 mRNA expression $(p=0.037$, Table 3$)$. Prior HCC treatment did not have a statistically significant impact.

\section{AFP response according to the intratumoral OCT1 mRNA expression}

For the evaluation of the AFP response, only patients with AFP levels $>20 \mathrm{ng} / \mathrm{ml}$ (AFP-positive HCC) were included in the analysis. Patients were only categorized as AFP responders if a reduction in AFP levels of at least $20 \%$ was achieved under treatment with sorafenib $[15,16]$. Table 4 shows the AFP response of the AFP positive patients in this cohort according to the OCT1 mRNA expression (<lower quartile vs. $\geq$ lower

Table 2 Cox regression

\begin{tabular}{llll}
\hline & HR & $95 \%-C l$ & $P$-value \\
\hline OCT1 expression level [log10] & 0.653 & $0.430-0.992$ & 0.046 \\
age (begin sorafenib) [years] & 0.980 & $0.955-1.007$ & 0.144 \\
\hline
\end{tabular}




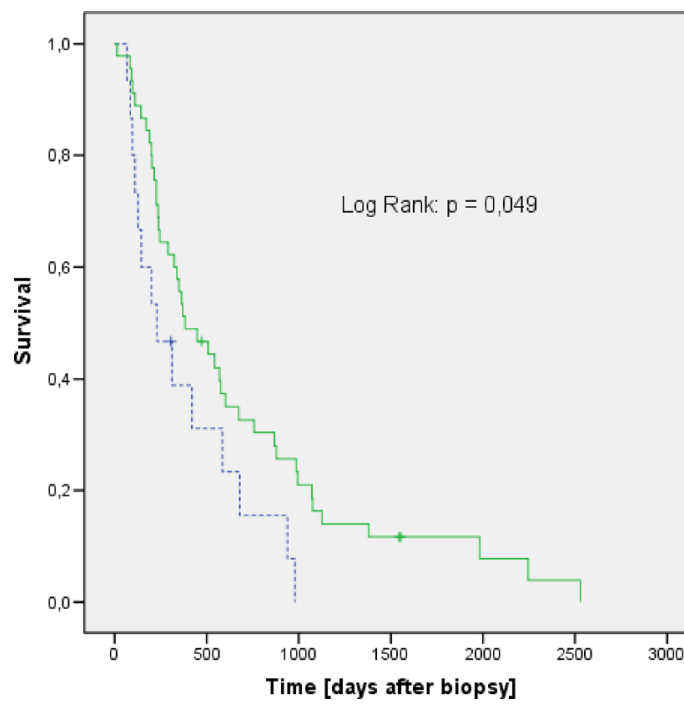

OCT1 high $(n=45)$

OCT1 low $(n=15)$

$+/+\quad$ censored according to a sensitivity analysis

quartile; $n=36$ ). Concerning the AFP response under treatment with sorafenib, there were no differences between the OCT1 mRNA low and the OCT1 mRNA high expression groups in this cohort $(p=0.633$, Table 4).

\section{Discussion}

Intratumoral downregulation of OCT1 in $\mathrm{HCC}$ has been described by us and others $[10,13]$. In a previous work we showed that down-regulation of OCT1 is associated with reduced survival in patients that underwent liver

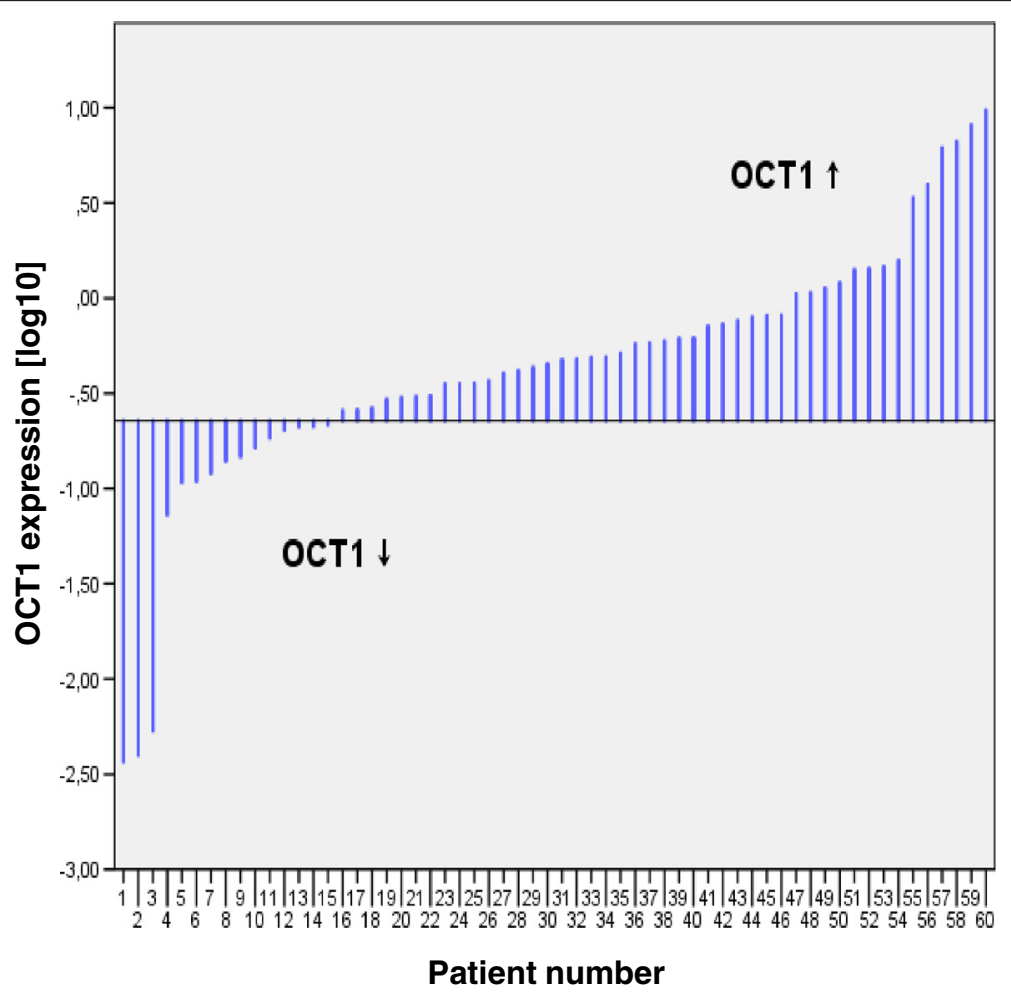

Fig. 2 Intratumoral OCT1 expression according to median. The patients were sorted by intratumoral OCT1 expression ( $n=60)$. Two patient groups were defined according to the lower quartile of intratumoral OCT1 expression 
Table 3 Patients and tumor characteristics according to the intratumoral OCT1 mRNA expression

\begin{tabular}{|c|c|c|c|c|}
\hline \multirow{3}{*}{\multicolumn{2}{|c|}{ Characteristics }} & OCT1 (SLC22A1) & OCT1 (SLC22A1) & $P$-value \\
\hline & & Low expression & High expression & \\
\hline & & ( $<$ lower quartile) & ( $\geq$ lower quartile) & \\
\hline \multicolumn{2}{|l|}{$n$} & 15 & 45 & \\
\hline \multicolumn{5}{|l|}{ Gender } \\
\hline \multicolumn{2}{|l|}{ male } & 14 & 40 & \\
\hline \multicolumn{2}{|l|}{ female } & 1 & 5 & 1.000(Fisher's exact test) \\
\hline \multicolumn{5}{|l|}{ Mean age } \\
\hline \multicolumn{2}{|c|}{ years (standard deviation) } & $65.022(7.190)$ & $64.658(11.667)$ & 0.910(unpaired $\mathrm{t}$ test) \\
\hline \multicolumn{5}{|c|}{ Underlying liver disease } \\
\hline \multicolumn{2}{|l|}{ alcohol } & 2 & 14 & \\
\hline \multicolumn{2}{|l|}{ HBV } & 3 & 8 & \\
\hline \multicolumn{2}{|l|}{$\mathrm{HCV}$} & 4 & 8 & \\
\hline \multicolumn{2}{|c|}{ steatosis or NASH } & 0 & 5 & \\
\hline \multicolumn{2}{|l|}{ others } & 5 & 6 & \\
\hline \multicolumn{2}{|l|}{ unknown } & 1 & 4 & 0.224(Fisher's exact test) \\
\hline \multicolumn{5}{|c|}{ Prior HCC treatment } \\
\hline \multicolumn{2}{|l|}{ yes } & 6 & 29 & \\
\hline \multicolumn{2}{|l|}{ no } & 9 & 16 & 0.133(Fisher's exact test) \\
\hline \multicolumn{5}{|c|}{ Tumor grading } \\
\hline \multicolumn{2}{|l|}{ G1 } & 3 & 10 & \\
\hline \multicolumn{2}{|l|}{$\mathrm{G} 2$} & 8 & 26 & \\
\hline \multicolumn{2}{|l|}{ G3 } & 4 & 4 & \\
\hline \multicolumn{2}{|l|}{ unknown } & 0 & 5 & 0.265(Mann-Whitney $U$ test) \\
\hline \multicolumn{5}{|c|}{ Tumor burden } \\
\hline \multirow[t]{2}{*}{ MVI } & absent & 4 & 27 & \\
\hline & present & 11 & 18 & 0.037(Fisher's exact test) \\
\hline \multirow[t]{2}{*}{ EHS } & absent & 5 & 12 & \\
\hline & present & 10 & 33 & 0.743(Fisher's exact test) \\
\hline $\mathrm{MVl}$ & & & & \\
\hline and/or EHS & absent & 1 & 5 & \\
\hline & present & 14 & 40 & 1.000(Fisher's exact test) \\
\hline BCLC classific & & & & \\
\hline A & & 0 & 1 & \\
\hline B & & 1 & 0 & \\
\hline C & & 12 & 38 & \\
\hline $\mathrm{D}$ & & 2 & 4 & \\
\hline unknown & & 0 & 2 & 0.988(Mann-Whitney $U$ test) \\
\hline ECOG PS & & & & \\
\hline 0 & & 2 & 9 & \\
\hline 1 & & 8 & 31 & \\
\hline 2 & & 2 & 3 & \\
\hline 3 & & 2 & 0 & \\
\hline unknown & & 1 & 2 & 0.099(Mann-Whitney U test) \\
\hline
\end{tabular}


Table 3 Patients and tumor characteristics according to the intratumoral OCT1 mRNA expression (Continued)

\begin{tabular}{|c|c|c|c|}
\hline \multicolumn{4}{|l|}{ Child-Pugh } \\
\hline A & 1 & 13 & \\
\hline B & 11 & 13 & \\
\hline C & 0 & 4 & \\
\hline unkown & 3 & 15 & 0.195(Mann-Whitney U test) \\
\hline \multicolumn{4}{|l|}{ Ascites } \\
\hline absent & 5 & 18 & \\
\hline present & 6 & 13 & \\
\hline unknown & 4 & 14 & 0.504(Fisher's exact test) \\
\hline \multicolumn{4}{|l|}{ Baseline AFP (ng/ml) } \\
\hline$\leq 20$ & 2 & 20 & \\
\hline$>20$ & 11 & 25 & \\
\hline unknown & 2 & 0 & 0.103(Fisher's exact test) \\
\hline \multicolumn{4}{|l|}{ mean duration } \\
\hline \multicolumn{4}{|l|}{ sorafenib treatment } \\
\hline days (standard deviation) & $161(126)$ & 149 (128) & 0.764(unpaired $t$ test) \\
\hline
\end{tabular}

resection or transplantation [10]. Whether reduced intratumoral OCT1 mRNA expression assessed from tumor biopsies is of prognostic value under sorafenib treatment has not been defined yet. We performed this retrospective study as the identification of novel biomarkers in HCC treatment is of special interest in terms of individualized medicine.

For this analysis, OCT1 mRNA was quantified with a commercially available primer set that has been comprehensively validated and correlated with OCT1 protein expression by our group [10, 17]. OCT1 exhibits SNPs that might affect OCT1 function. In the background of CML, several studies investigated the association between OCT1 SNPs and clinical outcome with contradictory results [18-21]. Importantly, one study suggests that contradictory results might be due to interference between SNPs and primer sites [19]. Upon request, the manufacturer of the primer assays used in this study ensured that the primer sites do not interfere with the most relevant SNPs as proposed by Giannoudis et al. [19]. A sensitivity analysis revealed that particularly patients with a baseline OCT1 mRNA expression within the range of the lower quartile have a significantly impaired survival under treatment with sorafenib. The poor prognosis under treatment might be at least in part explained by a reduced OCT1-mediated drug uptake due to non-functional, truncated proteins [13].

This retrospective analysis shows that a reduced intratumoral OCT1 mRNA expression results in a worse survival in patients treated with sorafenib. This effect is independent of other strong prognostic factors like the presence of ascites, BCLC stage and ECOG performance status [22]. A correlation between the prognostically unfavorable low intratumoral OCT1 expression and MVI could be shown here if the lower quartile of OCT1 expression was used as a cutoff $(p=0.037)$. This correlation is not significant if the cutoff is median OCT1 expression $(p=0.120$, data not shown). Also in previous studies using median OCT1 expression as a cutoff, a statistically significant correlation between OCT1 expression and MVI was not shown [10]. The impact of this observation will be further analyzed in a subsequent study.

The prognostic role of tumor markers like AFP in HCC has been studied extensively [23]. Previous studies showed that AFP response was significantly associated with the overall survival also in patients with advanced

Table 4 AFP response

\begin{tabular}{llll}
\hline Baseline AFP $(\mathrm{ng} / \mathrm{ml})>20$ & OCT1 (SLC22A1) & OCT1 (SLC22A1) & P-value \\
& $\begin{array}{l}\text { Low expression } \\
(<\text { lower quartile) }\end{array}$ & High expression & $(\geq$ lower quartile) \\
$\mathrm{n}$ & 11 & 25 & \\
\hline AFP responders & 1 & 6 & 14 \\
AFP non-responders & 7 & 5 & 0.633 (Fisher's exact test) \\
unknown & 3 & &
\end{tabular}


HCC treated with sorafenib [16]. Probably due to variable times of AFP measurement in this retrospective analysis we could not reproduce this finding in the context of OCT1 mRNA expression levels.

A limitation of the current study is the retrospective nature of data collection. The biopsies were acquired in context of primary diagnosis of the HCC. Variations in tumor genetics may occur during the course of disease [24]. In addition, the time frame between biopsy acquisition and beginning of sorafenib treatment varies. Due to this fact, a lead time bias and effects on the basis of variable stages of tumor spread should be considered [25]. Some patients enrolled in this analysis have been treated in the early phase after approval of sorafenib. Initially, few patients with reduced liver function and performance status were treated with sorafenib. To date, guidelines do not recommend the use of sorafenib in these patients [2]. As common for retrospective trials, the reliability and validity of patient's report in terms of adherence to medication remains unknown [26]. Radiological response could not be correlated with OCT1 mRNA expression levels in this cohort due to a lack of data.

Although the acquisition of HCC tissue via transcutaneous biopsy is a feasible method with a good riskbenefit ratio, it should be considered that intratumoral heterogeneity in OCT1 mRNA expression might occur. The alternative approach of a HCC resection remains reserved to a relatively small fraction of patients [2]. However, facing all the drawbacks, the identification of patient subgroups with the best response to an antitumor agent in HCC by information drawn from tumor biopsies is still a promising approach.

\section{Conclusions}

The identification of novel biomarkers for anticancer therapy is of particular importance in terms of prevention of side effects caused by therapeutics with limited efficacy in the individual patient as well as for economic reasons. This study shows that intratumoral OCT1 mRNA expression might play a role as a prognostic biomarker in sorafenib-based HCC therapy. Further, prospective trails are warranted on this topic.

\footnotetext{
Abbreviations

MVI: macrovascular invasion; EHS: extrahepatic spread; AFP: alpha-fetoprotein.

Competing interests

MAW: consulting and lecture fees from Bayer HealthCare and Bristol-Myers Squibb. PRG: receiving consulting and lecture fees from Bayer HealthCare, Bristol-Myers Squibb and Lilly. All other authors have no competing interests.
}

\section{Authors' contributions}

DG, TZ and PRG designed the study. DG and $J$ performed PCR analysis. WW and $\mathrm{MHL}$ participated in the statistical analysis. JV, FD, AL, SK, JMS, MAW, and AW participated in the acquisition and management of the clinical data. AS participated in the histological analysis. DG, $J$, and TZ wrote the manuscript. This publication contains essential parts of the dissertation of JL. All authors read and approved the final manuscript.

\section{Acknowledgements}

We thank Larissa Herbel for excellent technical assistance. This work was supported by an intramural funding of the University of Mainz (Inneruniversitäre Forschungsförderung Stufe I grant) to TZ.

\section{Author details}

${ }^{1} 1$ st Department of Medicine, University Medical Center Mainz, Langenbeckstr. 1, Mainz 55131, Germany. ${ }^{2}$ University Medical Center Mainz, Institute of Medical Biostatistics, Epidemiology and Informatics, Obere Zahlbacher Str. 69, Mainz 55131, Germany. ${ }^{3}$ Department of General-, Visceraland Transplantation Surgery, University Medical Center Mainz, Langenbeckstr. 1, Mainz 55131, Germany. ${ }^{4}$ Department of Pathology, University Medical Center Mainz, Langenbeckstr. 1, Mainz 55131, Germany.

Received: 15 July 2015 Accepted: 8 February 2016

Published online: 12 February 2016

\section{References}

1. El-Serag HB, Rudolph KL. Hepatocellular carcinoma: epidemiology and molecular carcinogenesis. Gastroenterology. 2007;132(7):2557-76. doi:10.1053/j.gastro.2007.04.061

2. EASL-EORTC clinical practice guidelines: management of hepatocellular carcinoma. J Hepatol. 2012;56(4):908-43. doi:10.1016/j.jhep.2011.12.001.

3. Jemal A, Bray F, Center MM, Ferlay J, Ward E, Forman D. Global cancer statistics. CA Cancer J Clin. 2011;61(2):69-90. doi:10.3322/caac.20107.

4. Asghar U, Meyer T. Are there opportunities for chemotherapy in the treatment of hepatocellular cancer? J Hepatol. 2012;56(3):686-95. doi:10.1016/j.jhep.2011.07.031.

5. Llovet JM, Ricci S, Mazzaferro V, Hilgard P, Gane E, Blanc JF, et al. Sorafenib in advanced hepatocellular carcinoma. N Engl J Med. 2008;359(4):378-90. doi:10.1056/NEJMoa0708857.

6. Cheng AL, Kang YK, Chen Z, Tsao CJ, Qin S, Kim JS, et al. Efficacy and safety of sorafenib in patients in the Asia-Pacific region with advanced hepatocellular carcinoma: a phase III randomised, double-blind, placebo-controlled trial. Lancet Oncol. 2009;10(1):25-34. doi:10.1016/s1470-2045(08)70285-7.

7. Camma C, Cabibbo G, Petta S, Enea M, lavarone M, Grieco A, et al. Costeffectiveness of sorafenib treatment in field practice for patients with hepatocellular carcinoma. Hepatology (Baltimore, Md). 2013;57(3):1046-54. doi:10.1002/hep.26221

8. Carr Bl, Carroll S, Muszbek N, Gondek K. Economic evaluation of sorafenib in unresectable hepatocellular carcinoma. J Gastroenterol Hepatol. 2010;25(11): 1739-46. doi:10.1111/j.1440-1746.2010.06404.x.

9. Shao YY, Hsu CH, Cheng AL. Predictive Biomarkers of Antiangiogenic Therapy for Advanced Hepatocellular Carcinoma: Where Are We? Liver cancer. 2013;2(2):93-107. doi:10.1159/000343845.

10. Heise M, Lautem A, Knapstein J, Schattenberg JM, Hoppe-Lotichius M, Foltys $D$, et al. Downregulation of organic cation transporters OCT1 (SLC22A1) and OCT3 (SLC22A3) in human hepatocellular carcinoma and their prognostic significance. BMC Cancer. 2012;12:109. doi:10.1186/1471-2407-12-109.

11. Lozano E, Herraez E, Briz O, Robledo VS, Hernandez-Iglesias J, GonzalezHernandez A, et al. Role of the plasma membrane transporter of organic cations OCT1 and its genetic variants in modern liver pharmacology. BioMed Res Int. 2013;2013:692071. doi:10.1155/2013/692071.

12. Minematsu T, Giacomini KM. Interactions of tyrosine kinase inhibitors with organic cation transporters and multidrug and toxic compound extrusion proteins. Mol Cancer Ther. 2011;10(3):531-9. doi:10.1158/1535-7163.mct-10-0731.

13. Herraez E, Lozano E, Macias Rl, Vaquero J, Bujanda L, Banales JM et al. The expression of SLC22A1 variants may affect the response of hepatocellular carcinoma and cholangiocarcinoma to sorafenib. Hepatology (Baltimore, Md). 2013. doi:10.1002/hep.26425

14. Nardinelli L, Sanabani SS, Didone A, Ferreira Pde B, Serpa M, Novaes MM, et al. Pretherapeutic expression of the hOCT1 gene predicts a complete molecular response to imatinib mesylate in chronic-phase chronic myeloid leukemia. Acta Haematol. 2012;127(4):228-34. doi:10.1159/000336610.

15. Chan SL, Mo FK, Johnson PJ, Hui EP, Ma BB, Ho WM, et al. New utility of an old marker: serial alpha-fetoprotein measurement in predicting radiologic response and survival of patients with hepatocellular carcinoma undergoing systemic chemotherapy. J Clin Oncol. 2009;27(3):446-52. doi:10.1200/jco.2008.18.8151. 
16. Personeni N, Bozzarelli S, Pressiani T, Rimassa L, Tronconi MC, Sclafani F, et al. Usefulness of alpha-fetoprotein response in patients treated with sorafenib for advanced hepatocellular carcinoma. J Hepatol. 2012;57(1):101-7. doi:10.1016/j.jhep.2012.02.016.

17. Lautem A, Heise M, Grasel A, Hoppe-Lotichius M, Weiler N, Foltys D, et al. Downregulation of organic cation transporter 1 (SLC22A1) is associated with tumor progression and reduced patient survival in human cholangiocellular carcinoma. Int J Oncol. 2013;42(4):1297-304.

18. Bazeos A, Marin D, Reid AG, Gerrard G, Milojkovic D, May PC, et al. hOCT1 transcript levels and single nucleotide polymorphisms as predictive factors for response to imatinib in chronic myeloid leukemia. Leukemia. 2010;24(6):1243-5.

19. Giannoudis A, Wang L, Jorgensen AL, Xinarianos G, Davies A, Pushpakom S, et al. The hOCT1 SNPs M420del and M408V alter imatinib uptake and M420del modifies clinical outcome in imatinib-treated chronic myeloid leukemia. Blood. 2013;121(4):628-37.

20. Kim DH, Sriharsha L, Xu W, Kamel-Reid S, Liu X, Siminovitch K, et al. Clinica relevance of a pharmacogenetic approach using multiple candidate genes to predict response and resistance to imatinib therapy in chronic myeloid leukemia. Clin Cancer Res. 2009;15(14):4750-8.

21. Maffioli M, Camos M, Gaya A, Hernandez-Boluda JC, Alvarez-Larran A, Domingo A, et al. Correlation between genetic polymorphisms of the hOCT1 and MDR1 genes and the response to imatinib in patients newly diagnosed with chronic-phase chronic myeloid leukemia. Leuk Res. 2011;35(8):1014-9.

22. Weinmann A, Koch S, Niederle IM, Schulze-Bergkamen H, Koniq J, HoppeLotichius M, et al. Trends in Epidemiology, Treatment, and Survival of Hepatocellular Carcinoma Patients Between 1998 and 2009: An Analysis of 1066 Cases of a German HCC Registry. J Clin Gastroenterol. 2013. doi:10.1097/MCG.0b013e3182a8a793.

23. Rich N, Singal AG. Hepatocellular carcinoma tumour markers: current role and expectations. Best Pract Res Clin Gastroenterol. 2014;28(5):843-53. doi:10.1016/.jppg.2014.07.018.

24. Gauthier A, Ho M. Role of sorafenib in the treatment of advanced hepatocellular carcinoma: An update. Hepatol Res. 2013;43(2):147-54. doi:10.1111/j.1872-034X.2012.01113.x.

25. Cucchetti A, Trevisani F, Pecorelli A, Erroi V, Farinati F, Ciccarese F et al. Estimation of lead-time bias and its impact on the outcome of surveillance for the early diagnosis of hepatocellular carcinoma. J Hepatol. 2014 doi:10.1016/j.jhep.2014.03.037.

26. Osterberg L, Blaschke T. Adherence to medication. N Engl J Med. 2005;353(5):487-97. doi:10.1056/NEJMra050100.

\section{Submit your next manuscript to BioMed Central and we will help you at every step:}

- We accept pre-submission inquiries

- Our selector tool helps you to find the most relevant journal

- We provide round the clock customer support

- Convenient online submission

- Thorough peer review

- Inclusion in PubMed and all major indexing services

- Maximum visibility for your research

Submit your manuscript at www biomedcentral.com/submit

) Biomed Central 